\title{
CUMPLIMIENTO NORMATIVO SOBRE FLUJOS DE EFECTIVO REPORTADOS POR EMPRESAS INDUSTRIALES BAJO NIIF
}

\author{
REGULATORY COMPLIANCE ON CASH FLOWS FOR INDUSTRIAL COMPANIES \\ REPORTED UNDER IFRS
}

\author{
Percy Antonio Vílchez Olivares * \\ Docente Asociado de la Facultad de Ciencias Contables \\ Universidad Nacional Mayor de San Marcos-UNMSM / Lima-Perú \\ [Recepción: Marzo de 2015/ Conformidad: Abril 2015]
}

\section{RESUMEN}

De acuerdo a las regulaciones contables emitidas en nuestro país por la Contaduría Pública de la Nación y la Superintendencia del Mercado de Valores, a partir del año 2011 se inició un proceso de adopción en forma obligatoria de las Normas Internacionales de Información Financiera (IFRS por sus siglas en inglés) emitidas por Junta de Normas de Contabilidad Financiera (IASB por sus siglas en inglés, organismo emisor de las Normas Internacionales de Información Financiera NIIF), para las empresas que tienen cotización bursátil, con excepción de las empresas bancarias, financieras y de seguros, y otras reguladas por la Superintendencia de Banca y Seguros.

El presente trabajo de investigación, tuvo por objetivo analizar el grado de cumplimiento de las empresas industriales que cotizan en la Bolsa de Valores de Lima en la adopción de las NIIF respecto a la presentación del Estado de Flujos de Efectivo y las revelaciones contables que se exigen en notas a los estados financieros respecto a los flujos de efectivo en el período 2014. Los resultados obtenidos demuestran que existe insuficiencia informativa y proporciona evidencia significativa de las categorías normativas relevantes sobre la información financiera de los flujos de efectivo que son afectados en el cumplimiento de las NIIF.

\section{Palabras clave:}

Efectivo; Normas Internacionales de Información Financiera; flujos de efectivo; cumplimiento normativo; usuarios de la información financiera.

\begin{abstract}
According to accounting regulations issued in Peru by the Public Accounting Office and the Superintendence of Securities, since 2011 a mandatory adoption process started of the International Financial Reporting Standards (IFRS) issued by the International Accounting Standards Board (IASB, issuing institution of the International Financial Reporting Standards- IFRS) for companies with share price, with the exception of banking, financial and insurance enterprises, and other regulated by the Superintendence of Banking and Insurance.

This research work analyzed the level of compliance of industrial companies listed on the Lima Stock Exchange in the adoption of IFRS regarding the presentation of the Cash Flow Statement and accounting disclosures required in notes to the financial statements regarding the cash flows in the period 2014. The results show that there is insufficient information and provides significant evidence of relevant normative categories over financial reporting of the cash flows that are affected in implementing IFRS.
\end{abstract}

\section{Keywords:}

Cash; International Financial Reporting Standards; cash flows; regulatory compliance; users of financial information.

\footnotetext{
* Magister en Ciencias Contables y Financieras - Universidad Diego Portales (Chile). E-mail: pvilchezcpa@gmail.com
} 


\section{INTRODUCCIÓN}

Las Normas Internacionales de Información Financiera (IFRS por sus siglas en inglés), son emitidas por la Junta de Normas Internacionales de Contabilidad (IASB por sus siglas en inglés), y constituyen estándares contables internacionales que basado en la constitución de la Fundación del Comité de Normas Internacionales de Contabilidad (por sus siglas en inglés IASCF), organismo supervisor del IASB tienen como objetivos desarrollar, buscando el interés público, un único conjunto de normas contables de carácter global que sean de alta calidad, comprensibles y de cumplimiento obligado, que requieran información de alta calidad, transparente y comparable en los estados financieros y en otros tipos de información financiera, para ayudar a los participantes en los mercados de capitales de todo el mundo, y a otros usuarios, a tomar decisiones económicas; promover el uso y la aplicación rigurosa de tales normas; cumplir lo anterior considerando las necesidades especiales de entidades pequeñas y medianas y de economías emergentes; y llevar a la convergencia entre las normas contables nacionales y las Normas Internacionales de Información Financiera, hacia soluciones de alta calidad.

La Fundación IFRS ha publicado un estudio en el 2015, en la que se señala que se "ha podido analizar 138 jurisdicciones de las 197 reconocidas por las Naciones Unidas, prácticamente en todas ellas, la autoridad competente se ha mostrado a favor de las Normas Internacionales de Información Financiera como la única norma de contabilidad global para la preparación y presentación de información financiera, de ahí que 126 jurisdicciones se han pronunciado positivamente, incluidos todos los países representados en el G20, que representan el 96 por ciento del Producto Bruto Interno mundial. Además de las declaraciones de intención, en 114 jurisdicciones $(82$ por ciento) el uso de las NIIF es obligatorio para todas o las mayorías de sus empresas con responsabilidad pública”. En ese sentido, existe un aumento progresivo hacia la utilización de las Normas Internacionales de Información Financiera.

Asimismo, de acuerdo con la lista Fortune 500, las NIIF son la norma contable utilizada por más del 52 por ciento de las empresas más grandes del mundo, mientras que los principios de contabilidad generalmente aceptados de los Estados Unidos ocupan el segundo lugar en utilización con el 29 por ciento de esas empresas.

Por otro lado, en el Perú, se inició a partir del año 2010 un proceso de adopción hacia las Normas Internacionales de Información Financiera. Al respecto, el Consejo Normativo de Contabilidad ha emitido la Resolución No 045-2010-EF/94 del 25.11.10. sobre la aplicación de las NIIF Completas para empresas que tienen ingresos o activos mayores a 3,000 unidades impositivas tributarias y la CONASEV (hoy Superintendencia del Mercado de Valores) ha emitido la Resolución No 102-2010-EF/94.01.1 del 14.10.10 que establece que las sociedades emisoras de valores inscritos en el Registro Público del Mercado de Valores, con excepción de las empresas bancarias, financieras y de seguros, y otras reguladas por la $\mathrm{Su}$ perintendencia de Banca y Seguros, deberán preparar sus estados financieros con observancia plena de las NIIF que emita el IASB vigentes internacionalmente, de acuerdo con dicha norma los primeros estados financieros que deben formular las empresas aplicando plenamente las NIIF serán los correspondientes a la información financiera auditada anual al 31 de diciembre del 2011; esta obligación implica que estas entidades deben aplicar estos estándares contables internacionales en forma estricta y sin reservas.

En ese sentido, las entidades deben informar que emiten los estados financieros de acuerdo a los parámetros establecidos en las Normas Internacionales de Información Financiera. Esto significa que las entidades deben cumplir con todos los requerimientos de todas las normas contables aplicables. Sin embargo, el requisito universal es que los estados financieros deben presentar en forma razonable la situación financiera, el rendimiento financiero y los flujos de efectivo de una entidad. La aplicación correcta de las NIIF, con la revelación adicional cuando es requerida, se presume que resulta en una emisión de estados financieros que logran una presentación razonable de toda la situación financiera y económica de la entidad.

De acuerdo a este nuevo marco normativo internacional las Normas Internacionales de Información 
Financiera deben ser aplicadas en la presentación de estados financieros con propósito general. Los estados financieros con propósito general son aquellos elaborados para satisfacer las necesidades de usuarios que no están en una posición de demandar informes que estén hechos a la medida para cumplir sus necesidades específicas de información. Ellos incluyen estados financieros presentados individualmente o aquellos que se presenten dentro de otro documento público, tal como un informe o memoria anual.

El objetivo de los estados financieros con propósitos generales es suministrar información acerca de la situación financiera, del rendimiento financiero y de los flujos de efectivo de una entidad, información que es útil a una gran variedad de usuarios para tomar decisiones económicas.

El Estado de Flujos de Efectivo proporciona información que permite a los usuarios evaluar los cambios en los activo netos de una entidad, su estructura financiera (incluyendo su liquidez y solvencia) y su capacidad para afectar a los importes y fechas de los flujos de efectivo, a fin de adaptarse a la evolución de las circunstancias y oportunidades.

De acuerdo a la NIC 7 en su párrafo 3 se señala "Los usuarios de los estados financieros están interesados en saber cómo la entidad genera y utiliza el efectivo y los equivalentes al efectivo. Esta necesidad es independiente de la naturaleza de las actividades de la entidad, incluso cuando el efectivo pueda ser considerado como el producto de la entidad en cuestión, como puede ser el caso de las entidades financieras. Básicamente, las entidades necesitan efectivo por las mismas razones, por muy diferentes que sean las actividades que constituyen su principal fuente de ingresos. En efecto, todas ellas necesitan efectivo para llevar a cabo sus operaciones, pagar sus obligaciones y suministrar rendimientos a sus inversores". Por consiguiente, esta norma exige que todo conjunto de "Estados Financieros" debe incluir un Estado de Flujos de Efectivo de forma obligatoria para las entidades.

Desde la perspectiva del paradigma de la utilidad de la información contable, el Estado de Flujos de Efectivo debe proporcionar información pertinente de los cambios en los flujos de efectivo en un período determinado que sirva para la toma de decisiones económicas. Es necesario validar si la información financiera de los flujos de efectivo emitidos por las empresas industriales que cotizan en la Bolsa de Valores de Lima en el período 2014 cumple con el requerimiento normativo indicado en las NIIF. La investigación se realiza aplicando un análisis cualitativo de esta situación.

El presente trabajo de investigación incluye una introducción, se proporciona un marco teórico y normativo, a continuación se presentan las principales características del desarrollo metodológico. Posteriormente, se presentan los resultados que se desprenden del análisis cualitativo que son la base para emitir las conclusiones de la investigación.

\section{MARCO TEÓRICO}

\section{Información del Estado de Flujos de Efectivo para los Usuarios de la Información Financiera}

La NIC 7 fue emitida originalmente en el año 1977 con el nombre "Estado de Cambios en la Situación Financiera”, la misma que tenía como tema central los cambios en el capital de trabajo de un período a otro, esta información era muy requerida en esos tiempos y coincidía con la literatura contable existente en autores americanos como Ralph Dale Kennedy en su texto Estados Financieros y Robert Anthony en su texto la Contabilidad en la Administración de Empresas, que difundían los estados de movimientos de fondos desde la óptica del capital de trabajo. Pero desde el año 1992 se modificó la NIC 7 con el nombre "Estado de Flujos de Efectivo", es decir, ahora se concentra en los cambios en el dinero en efectivo y en los equivalentes de efectivo.

Asimismo, diversos organismos reguladores contables a nivel internacional desde la década de los ochenta del siglo XX, han incorporado a su normatividad contable el Estado de Flujos de Efectivo, por ejemplo el organismo emisor FASB de Estados Unidos emitió en 1987 la norma SFAS N 95 "Estado de Flujos de Efectivo" que establece normas para informar sobre flujos de efectivo en los Estados Unidos, esta norma reemplaza a la Opinión APB N 19 "Estado de Cambios en la Situación Financiera" (esta norma fue emitida en 1971 por la Junta 
de Principios de Contabilidad (APB) del Instituto Americano de Contadores Públicos Certificados requiriendo presentar un Estado de Cambios en la Situación Financiera como un estado financiero básico), y requiere un Estado de Flujos de Efectivo como parte de los estados financieros según los Principios de Contabilidad Generalmente Aceptados para todas las empresas de negocios, en lugar del Estado de Cambios en la Situación Financiera.

Hace unas décadas y en la actualidad, la regulación contable internacional se ha dirigido al análisis de los fondos de las empresas hacia el flujo de efectivo; en ese sentido, la información financiera incluida en el Estado de Flujos de Efectivo permite obtener información financiera valiosa sobre los ingresos por cobros en efectivo y egresos por pagos en efectivo de una entidad en un período económico. Adicionalmente, este estado financiero presenta información sobre el comportamiento del efectivo en las actividades de operación, inversión y financiación de la entidad durante el periodo. Por lo tanto, un Estado de Flujos de Efectivo debe ayudar a los inversionistas, prestamistas, acreedores y a otros usuarios de la información financiera en la evaluación de factores tales como:

a) Capacidad de la entidad de generar flujos de efectivo positivos en períodos futuros.

b) Capacidad de la entidad de cumplir con sus obligaciones y pagar dividendos.

c) Necesidad de la entidad de obtener financiación externa.

d) Aspectos de las transacciones de inversión y financiación de la empresa que hacen uso de efectivo y las que no hacen uso de este durante el período.

e) Causas del cambio de efectivo y equivalentes de efectivo entre el inicio y el final del período contable.

Asimismo, un Estado de Flujos de Efectivo ayuda a los usuarios de los estados financieros a evaluar la capacidad de la entidad de tener efectivo suficiente, tanto en el corto plazo como en el largo plazo. Por esta razón, el Estado de Flujos de Efectivo es útil prácticamente para todos los interesados en la salud financiera y económica de la entidad: los inversio- nistas, los prestamistas, los acreedores, la gerencia y los competidores actuales y potenciales.

Finalmente, la NIC 1 "Presentación de Estados Financieros" en el párrafo 111 señala "La información sobre los flujos de efectivo proporciona a los usuarios de los estados financieros una base para evaluar la capacidad de la entidad para generar efectivo y equivalentes al efectivo y las necesidades de la entidad para utilizar esos flujos de efectivo. La NIC 7 establece los requerimientos para la presentación y revelación de información sobre flujos de efectivo".

\section{Presentación del Estado de Flujos de Efectivo según la NIC 7 Estado de Flujos de Efectivo}

El Consejo de Normas Internacionales de Contabilidad (IASB) en el 2001 adoptó la NIC 7 Estado de Flujos de Efectivo, que había sido originalmente emitida por el Comité de Normas Internacionales de Contabilidad en 1992, posteriormente esta norma ha tenido actualizaciones como resultado de los cambios en la terminología utilizada en las NIIF procedentes de requerimientos de la NIC 1 Presentación de Estados Financieros (emitida por el IASB en el 2007), el título de la NIC 7 se sustituyó por Estado de Flujos de Efectivo. Asimismo, otras NIIF han realizado modificaciones de menor importancia en esta norma, estas incluyen la NIIF 10 Estado Financieros Consolidados (emitida en el 2011), la NIIF 11 Acuerdos Conjuntos (emitida en el 2011) y el documento Entidades de Inversión (modificaciones a las NIIF 10, NIC 12 y NIC 27 emitida en el 2012).

El objetivo de la NIC 7 es requerir el suministro de información sobre los cambios históricos en el efectivo y equivalentes al efectivo de una entidad mediante un Estado de Flujos de Efectivo en el que los flujos de fondos del período se clasifiquen según si proceden de actividades de operación, de inversión o de financiación.

La NIC 7 define el término efectivo y señala que comprende tanto el efectivo como los depósitos bancarios a la vista y el término equivalentes al efectivo y señala que comprende las inversiones a corto plazo de gran liquidez, que son fácilmente convertibles en importes determinados de efectivo, estando sujetos a un riesgo poco significativo de cambios en su valor; 
los equivalentes al efectivo, se tienen, más que para propósitos de inversión o similares, para cumplir los compromisos de pago a corto plazo.

De acuerdo a la NIC 7 en su párrafo 10 se señala "El estado de flujos de efectivo informará acerca de los flujos de efectivo habidos durante el período, clasificándolos por actividades de operación, de inversión, y de financiación".

Actividades de operación "son las actividades que constituyen la principal fuente de ingresos de actividades ordinarias de la entidad, así como otras actividades que no pueden ser calificadas como de inversión o financiación”. (NIC 7 párrafo 6).

El importe de los flujos de efectivo procedentes de actividades de operación es un indicador clave de la medida en la cual estas actividades han generado fondos líquidos suficientes para amortizar las financiaciones, mantener la capacidad de operación de la entidad, pagar dividendos y realizar nuevas inversiones sin recurrir a fuentes externas de financiación. Estos flujos proceden de las operaciones y otros hechos que se incluyen en la determinación de los resultados del período. En resumen los flujos de efectivo por actividades de operación incluyen:

a) Cobros por las ventas de bienes y la prestación de servicios;

b) Cobros por regalías, cuotas, comisiones y otros ingresos;

c) Pagos a proveedores por el suministro de bienes y servicios;

d) Pagos a y por cuenta de los empleados;

e) Cobros y pagos de las entidades de seguros por primas y prestaciones, anualidades y otras obligaciones derivadas de las pólizas suscritas;

f) Pagos o devoluciones de impuestos sobre las ganancias, a menos que puedan clasificarse como actividades de inversión o financiación; y

g) Cobros y pagos derivados de contratos que se tienen para intermediación o para negociar con ellos.

Actividades de inversión "son las actividades vinculadas a la adquisición y disposición de activos a largo plazo, así como de otras inversiones no incluidas en el efectivo y los equivalentes al efectivo". (NIC 7 párrafo 6).
El importe de los flujos de efectivo procedentes de actividades de inversión representan la medida en la cual la entidad ha efectuado desembolsos para recursos que se estima van a producir ingresos y flujos de efectivo en el futuro. En ese sentido, solo los desembolsos que generan un reconocimiento de un activo en el estado de situación financiera cumplen las condiciones para que se clasifique como actividades de inversión. En resumen los flujos de efectivo por actividades de inversión incluyen:

a) Pagos por la adquisición de propiedades, planta y equipo, activos intangibles y otros activos a largo plazo.

b) Cobros por ventas de propiedades, planta y equipo, activos intangibles y otros activos a largo plazo;

c) Pagos por la adquisición de instrumentos de pasivo o de patrimonio, emitidos por otras entidades, así como participaciones en negocios conjuntos;

d) Cobros por venta y reembolso de instrumentos de pasivo o de capital emitidos por otras entidades, así como participaciones en negocios conjuntos;

e) Anticipos de efectivo y préstamos a terceros (distintos de las operaciones de ese tipo hechas por entidades financieras);

f) Cobros derivados del reembolso de anticipos y préstamos a terceros (distintos de las operaciones de este tipo hechas por entidades financieras);

g) Pagos derivados de contratos a término, de futuro, de opciones y de permuta financiera, excepto cuando dichos contratos se mantengan por motivos de intermediación u otros acuerdos comerciales habituales, o bien cuando los anteriores pagos se clasifiquen como actividades de financiación; y

h) Cobros procedentes de contratos a término, a futuro, de opciones y de permuta financiera, excepto cuando dichos contratos se mantengan por motivos de intermediación u otros acuerdos comerciales habituales, o bien cuando los anteriores cobros se clasifiquen como actividades de financiación.

Actividades de financiación "son las actividades que producen cambios en el tamaño y composición de los capitales propios y de los préstamos tomados por parte de la entidad”. (NIC 7 párrafo 6). 
El importe de los flujos de efectivo procedentes de actividades de financiación representan las bases para realizar proyecciones de necesidades de efectivo para cubrir compromisos con los suministradores de capital a la entidad. En resumen los flujos de efectivo por actividades de financiación incluyen:

a) Cobros por emisión de acciones $u$ otros instrumentos de capital;

b) Pagos a los propietarios por adquirir o rescatar las acciones de la entidad;

c) Cobros por la emisión de obligaciones sin garantía, préstamos, bonos, cédulas hipotecarias y otros fondos tomados en préstamo, ya sea a largo o a corto plazo;

d) Reembolsos en efectivo de fondos tomados en préstamo; $y$

e) Pagos realizados por arrendatario financiero.

Para informar los flujos de efectivos de las actividades de operación se puede usar el método directo y el método indirecto, pero se debe utilizar preferentemente el método directo (ver NIC 7 párrafo 19), en donde se presentan por separado las principales categorías de cobros y pagos en términos brutos. Alternativamente, se puede utilizar el método indirecto, procedimiento por el cual se comienza con la ganancia o pérdida, corrigiendo luego los efectos de las transacciones no monetarias, por todo tipo de partidas de pago diferido y acumulaciones (o devengos) que son la causa de cobros y pagos en el pasado o en el futuro, así como de las partidas de pérdidas o ganancias asociadas con flujos de efectivo de operaciones clasificadas como de inversión o financiación.

Respecto a la presentación en el Estado de Flujos de Efectivo las actividades de inversión y financiación utilizando el método directo y el método Indirecto, se presentan en forma similar.

Respecto al contenido del Estado de Flujos de Efectivo, la Superintendencia del Mercado de Valores (SMV por sus abreviaturas) de Perú ha diseñado un formato para su elaboración por el método directo y otro por el método indirecto, el mismo que esta concordado con lo planteado por las NIIF. Para acceder a los formatos estructurados por la SMV para los nuevos estados financieros bajo NIIF de empresas supervisadas por la SMV, y en especial el formato del Estado de Flujos de Efectivo, deberá visitar su sistio web ${ }^{1}$.

\section{Intereses y dividendos}

La NIC 7 en su párrafo 31 señala "Los flujos de efectivo correspondientes tanto a los intereses recibidos y pagados como a los dividendos percibidos y satisfechos deben ser revelados por separado. Cada una de las anteriores partidas debe ser clasificada de forma coherente, periodo a periodo, como perteneciente a actividades de operación, de inversión o de financiación". Respecto, a los intereses la norma señala que la entidad debe revelar los intereses pagados durante un período en el estado de flujos de efectivo, independientemente si ha sido reconocido como gasto en el resultado del período o si se capitalizó los intereses de acuerdo con la NIC 23 Costos por Préstamos.

Respecto a la clasificación de los intereses pagados, así como los intereses y dividendos cobrados no existe consenso para su clasificación en las entidades no financieras, en ese sentido, pueden ser clasificados como flujos de efectivo por actividades de operación $y$ de forma alternativa pueden clasificarse como flujos de efectivo por actividades de inversión o financiación respectivamente.

Por otro lado, los dividendos pueden clasificarse como flujos de efectivo de actividades de financiación, pero alternativamente pueden ser clasificados como componentes de los flujos de efectivo de las actividades de operación.

\section{Impuesto a las ganancias}

La NIC 7 en su párrafo 35 señala "Los flujos de efectivo procedentes de pagos relacionados con el impuesto a las ganancias deben revelarse por separado, y deben ser clasificados como flujos de efectivo procedentes de actividades de operación, a menos que puedan ser específicamente asociados con actividades de inversión o de financiación”. Sobre este párrafo, debo precisar que en caso de distribuir el pago por impuestos entre más de un tipo de actividad,

1 http://www.smv.gob.pe/Frm_PlantillaInfFin.aspx?data.

196/ QVIPURAMAYOC | Vol. 23(43) 2015 
se informará también del importe total de impuestos pagados en el período.

\section{Transacciones no monetarias}

La NIC 7 en su párrafo 43 señala "Las transacciones de inversión o financiación que no han requerido el uso de efectivo o equivalentes al efectivo se excluirán del Estado de Flujos de Efectivo. Estas transacciones deben ser objeto de información, en cualquier otra parte dentro de los estados financieros, de manera que suministren toda la información relevante acerca de tales actividades de inversión o financiación". Es claro que muchas de las actividades que realiza una entidad no tienen un impacto directo en los flujos de efectivo del período, por lo que no deben revelarse en el Estado de Flujos de Efectivo, por ejemplo: la conversión de deuda en patrimonio, la revaluación de activos inmovilizados, etc.

\section{Componentes de las partidas de efectivo y equi- valentes al efectivo}

La NIC 7 en su párrafo 45 señala "Una entidad revelará los componentes del efectivo y equivalentes al efectivo, y presentará una conciliación de los importes de su estado de flujos de efectivo con las partidas equivalentes sobre las que se informa en el estado de situación financiera”. Además, la entidad debe revelar los criterios adoptados para determinar la composición de las partidas de efectivo y equivalentes al efectivo, de acuerdo con lo previsto en la NIC 1 Presentación de Estados Financieros.

\section{Otra información a revelar}

La NIC 7 en su párrafo 48 señala "La entidad debe revelar en sus estados financieros, acompañado de un comentario por parte de la gerencia, cualquier importe significativo de sus saldos de efectivo y equivalentes al efectivo que no esté disponible para ser utilizado por ella misma o por el grupo al que pertenece”. Lo anterior, se explica porque algunas entidades tienen fondos restringidos por asuntos legales o por disposi- ciones de gobierno en la restringe el uso de los fondos a actividades específicas.

\section{DESARROLLO METODOLÓGICO}

\section{Diseño Metodológico}

Para dar respuesta al problema de investigación se diseña una estrategia metodológica cualitativa para producir datos descriptivos y que en esencia es inductiva e interpretativa de los hechos a investigar. A continuación, se presenta los lineamientos estratégicos en el diseño metodológico de esta investigación.

\section{Determinación de la Muestra de Estudio}

La población de la cual se obtuvo una muestra representativa está constituida por 264 empresas que tienen cotización bursátil en la Bolsa de Valores de Lima y que reportan información financiera anual a la Superintendencia del Mercado de Valores en el período 2014. En la actualidad no todas las empresas que tienen cotización bursátil reportan información financiera bajo Normas Internacionales de Información Financiera por regulación contable especial y otras que debiendo presentar información financiera bajo Normas Internacionales de Información Financiera no lo realizan por alguna circunstancia.

La muestra se determinó mediante muestreo no probabilístico discrecional, el cual corresponde a un grupo de empresas individuales industriales cotizadas en el mercado bursátil, que han adoptado las normas NIIF, y se consideró que los documentos a analizar son la información financiera auditada, el cual incluye dictamen de los auditores, estados financieros y las notas a los estados financieros que han sido sujetos a auditoría financiera en el período 2014.

De las 264 empresas cotizadas en la Bolsa de Valores que presentaron estados financieros en el año 2014, se han considerado 41 empresas (15.53\%) y que corresponden a empresas industriales y que han presentado información financiera de flujos de efectivo bajo Normas Internacionales de Información Financiera (Ver Cuadro $\mathrm{N}^{\circ} 01$ ). 
Cuadro No 01: Determinación de la Muestra Utilizada

\begin{tabular}{|l|r|r|}
\hline \multicolumn{1}{|c|}{ Grupo de Empresas } & $\begin{array}{c}\text { Número de } \\
\text { empresas }\end{array}$ & $\begin{array}{c}\text { \% de } \\
\text { participación }\end{array}$ \\
\hline Empresas registradas para presentar estados financieros & 264 & $100.00 \%$ \\
\hline $\begin{array}{l}\text { Empresas de los sectores agrario, minero, de servicios públicos y } \\
\text { diversas }\end{array}$ & -156 & $-59.09 \%$ \\
\hline $\begin{array}{l}\text { Empresas que no aplican IFRS por corresponder a Bancos, Cias. de } \\
\text { Seguros, AFP; y otras similares }\end{array}$ & -63 & $-23.86 \%$ \\
\hline $\begin{array}{l}\text { Empresas industriales en liquidación o presentan información bajo } \\
\text { principios de contabilidad generalmente aceptados }\end{array}$ & -4 & $-1.52 \%$ \\
\hline =Total Muestra Seleccionada & $\mathbf{4 1}$ & $\mathbf{1 5 . 5 3 \%}$ \\
\hline
\end{tabular}

Fuente: Elaboración Propia.

Las 41 empresas industriales que presentan información financiera auditada para período 2014 que se analizaron, se muestra en el Cuadro $\mathrm{N}^{\circ} 02$, dicha información fueron descargadas desde el repositorio de información de la Bolsa de Valores de Lima.

Cuadro No 02: Empresas Industriales Observadas

\begin{tabular}{|c|c|c|c|}
\hline $\mathbf{N}^{\circ}$ & Nombre & $\mathbf{N}^{\circ}$ & Nombre \\
\hline 1. & Agroindustrias AIB S.A. & 22. & Industria Textil Piura S.A. \\
\hline 2. & Agrícola y Ganadera Chavin de Huantar S.A. & 23. & Industrias del Envase S.A. \\
\hline 3. & Alicorp S.A.A. & 24. & Industrias Electro Químicas S.A. \\
\hline 4. & Austral Group S.A.A. & 25. & Intradevco Industrial S.A. \\
\hline 5. & Cementos Pacasmayo S.A.A. & 26. & Laive S.A. \\
\hline 6. & Cervecería San Juan S.A. & 27. & Lima Caucho S.A. \\
\hline 7. & Compañía Goodyear del Perú S.A. & 28. & Manufactura de Metales y Aluminio Record S.A. \\
\hline 8. & Compañía Universal Textil S.A. & 29. & Metalúrgica Peruana S.A. \\
\hline 9. & Consorcio Industrial de Arequipa S.A. & 30. & Michell y Cïa S.A. \\
\hline 10. & Corporación Aceros Arequipa S.A. & 31. & Motores Diesel Andinos S.A. \\
\hline 11. & Corporación Cerámica S.A. & 32. & Peruana de Moldeados S.A. \\
\hline 12. & Corporación Lindley S.A. & 33. & Pesquera Exalmar S.A.A. \\
\hline 13. & Creditex S.A.A. & 34. & Petróleos del Perú Petroperú S.A. \\
\hline 14. & Empresa Editora el Comercio S.A. & 35. & Quimpac S.A. \\
\hline 15. & Empresa Siderúrgica del Perú S.A.A. & 36. & Reactivos Nacionales S.A. \\
\hline 16. & Exsa S.A. & 37. & Refinería la Pampilla S.A. \\
\hline 17. & Fábrica Nacional de Acumuladores Etna S.A. & 38. & Sociedad Industrial de Artículos de Metal S.A.C. \\
\hline 18. & Fábrica Peruana Eternit S.A. & 39. & Unión de Cerveceras Peruanas Backus y Johnston S.A.A. \\
\hline 19. & Gloria S.A. & 40. & Unión Andina de Cementos S.A.A. \\
\hline 20. & Hidrostal S.A. & 41. & Yura S.A. \\
\hline 21. & Indeco S.A. & & \\
\hline
\end{tabular}

Fuente: Bolsa de Valores de Lima: http://www.bvl.com.pe/mercempresas.html. Elaboración Propia. 


\section{Objeto de Estudio}

El presente análisis tiene por objetivo identificar el tipo de método de presentación del Estado de Flujos de Efectivo y el grado de cumplimiento de las empresas industriales respecto a la regulación contable de las Normas Internacionales de Información Financiera en la presentación de información financiera anual y en particular en el Estado de Flujos de Efectivo y sus revelaciones contables respecto a los flujos de efectivo en el período 2014. Por lo anterior, en el Cuadro $\mathrm{N}^{\circ} 03$ se identifican las 4 categorías utilizadas para desarrollar el análisis cualitativo propuesto en esta investigación.

Cuadro No 03: Clasificación del Objeto de Estudio

\begin{tabular}{|c|l|l|}
\hline $\begin{array}{c}\text { Categoría } \\
\text { de Tipo }\end{array}$ & \multicolumn{1}{|c|}{ Descripción } & \multicolumn{1}{|c|}{ Fuente } \\
\hline 1 & Elección del método de presentación del estado de flujos de efectivo & $\begin{array}{l}\text { Estado de Flujos de } \\
\text { Efectivo }\end{array}$ \\
\hline 2 & $\begin{array}{l}\text { Omisiones en la revelación de intereses dividendos e impuesto a las } \\
\text { ganancias }\end{array}$ & $\begin{array}{l}\text { Estado de Flujos de } \\
\text { Efectivo }\end{array}$ \\
\hline 3 & $\begin{array}{l}\text { Omisiones en las descripciones en notas explicativas de criterios y } \\
\text { políticas contables del efectivo }\end{array}$ & $\begin{array}{l}\text { Notas a los estados } \\
\text { financieros }\end{array}$ \\
\hline 4 & $\begin{array}{l}\text { Omisiones en la descripción en estados financieros o notas explicativas } \\
\text { sobre los componentes del efectivo, transacciones no monetarias, y } \\
\text { grado de disponibilidad. }\end{array}$ & $\begin{array}{l}\text { Notas a los estados } \\
\text { financieros }\end{array}$ \\
\hline
\end{tabular}

Fuente: Elaboración Propia.

En esta investigación cualitativa se busca describir las principales orientaciones que subyacen en los contenidos de los cuatro tipos de observaciones que se identificaron en la información financiera sobre los flujos de efectivo. La técnica que se utilizó se basó en el Análisis de Contenido.

\section{RESULTADOS}

A continuación se detalla el análisis de contenido de las fuentes de información que sustentan los diversos tipos de categorías de información vinculados a los estados financieros anuales por el período 2014, además se señalan las principales orientaciones identificadas en el análisis de las categorías.

\section{Análisis a la Categoría de Tipo 1}

La categoría de tipo 1 está definida como los métodos de presentación de estados financieros del Estado de Flujos de Efectivo definidos bajo Normas Internacionales de Información Financiera. En ese sentido, de la información presentada en el Cuadro $\mathrm{N}^{\circ} 04$ se concluye que el método de presentación más utilizado en la elaboración del Estado de Flujos de Efectivo es el método directo con un $82.93 \%$, mientras que el uso del método indirecto es el menos frecuente con un $17.07 \%$.

Cuadro No 04: Número de Empresas en Función al Método de Presentación del Estado de Flujos de Efectivo en el período 2014

\begin{tabular}{|l|c|c|}
\hline \multicolumn{1}{|c|}{ Métodos de Presentación } & Frecuencia & $\begin{array}{c}\text { Relación sobre Total de Estados de } \\
\text { Flujos de Efectivo observados } \\
\text { Diciembre 2014 (N=41) }\end{array}$ \\
\hline Estado de Flujos de Efectivo- método directo & 34 & $82.93 \%$ \\
\hline Estado de Flujos de Efectivo- método indirecto & 7 & $17.07 \%$ \\
\hline
\end{tabular}

Fuente: Elaboración Propia. 
Los resultados obtenidos indican la preferencia por el uso del método directo que coincide con el grado de preferencia indicado en las NIIF. Debo precisar, que el método directo es un método disponible de utilizar en forma alterna.

\section{Análisis a la Categoría de Tipo 2}

La categoría de tipo 2 está definida como las omisiones en la revelación de los intereses, dividendos e impuesto a las ganancias, que son exigidos por la NIC 7. En ese sentido, de la información presentada en el Cuadro $\mathrm{N}^{\circ} 05$ presenta las omisiones en la revelación de partidas en el Estado de Flujos de Efectivo, siendo la partida concerniente a los intereses cobrados la que tiene el menor nivel de cumplimiento con un $22.22 \%$ ( 28 empresas incumplen lo requerido), luego comente en orden de mayor a menor incumplimiento le siguen los pagos por impuestos a las ganancias con un nivel de cumplimiento del $42.50 \%$ (23 empresas incumplen lo requerido), los intereses pagados con un nivel de cumplimiento del 58.54\% (17 empresas incumplen lo requerido), los dividendos cobrados con un nivel de cumplimiento del $90.00 \%$ (1 empresa incumple lo requerido) y los dividendos pagados con un nivel de cumplimiento del 96.67\% (1 empresa incumple lo requerido).

Cuadro No 05: Cumplimiento en la Revelación de Partidas en el Estado de Flujos de Efectivo en el período 2014

\begin{tabular}{|l|r|r|r|r|r|}
\hline \multicolumn{1}{|c|}{ Contenido } & $\begin{array}{c}\text { Intereses } \\
\text { Cobrados }\end{array}$ & $\begin{array}{c}\text { Intereses } \\
\text { Pagados }\end{array}$ & $\begin{array}{c}\text { Dividendos } \\
\text { Cobrados }\end{array}$ & $\begin{array}{c}\text { Dividendos } \\
\text { Pagados }\end{array}$ & $\begin{array}{c}\text { Impuesto } \\
\text { Ganancias } \\
\text { Pagados }\end{array}$ \\
\hline Número de empresas analizadas & 41 & 41 & 41 & 41 & 41 \\
\hline Número de empresas que incurren en la partida & 36 & 41 & 0 & 31 & 11 \\
\hline $\begin{array}{l}\text { Número de empresas que no incurren en la } \\
\text { partida }\end{array}$ & 5 & 8 & 24 & 9 & 17 \\
\hline $\begin{array}{l}\text { Número de empresas que reportan correctamen- } \\
\text { te la partida en el Estado de Flujos de Efectivo }\end{array}$ & & & & 29 \\
\hline $\begin{array}{l}\text { Relación de cumplimiento de la revelación con- } \\
\text { table de la partida sobre la partida incurrida }\end{array}$ & $22.22 \%$ & $58.54 \%$ & $90.00 \%$ & $96.67 \%$ & $42.50 \%$ \\
\hline
\end{tabular}

Fuente: Elaboración Propia.

Los resultados obtenidos indican que existe información insuficiente respecto al requerimiento de revelaciones exigidas por la NIC 7, esta situación no significa que los saldos y totales de las diversas partidas de los Estados de Flujos de Efectivo de las empresas que reportaron información financiera sean incorrectos.

\section{Análisis a la Categoría de Tipo 3}

La categoría de tipo 3 está definida como las omisiones en las descripciones en notas a los estados financieros de los criterios y políticas contables adoptadas en la composición de las partidas del efectivo y equivalentes de efectivo definidos bajo NIIF. En ese sentido, de la información presentada en el Cuadro $\mathrm{N}^{\circ} 06$ se concluye que existe un gran número de empresas que cumplen con informar los criterios y políticas contables del efectivo, el nivel cumplimiento es del $85.36 \%$ (35 empresas cumplen con lo requerido) y el nivel de incumplimiento es del $14.64 \%$ (6 empresas no cumplen con lo requerido). 
Cuadro No 06: Cumplimiento en la Revelación de Políticas Contables sobre el Efectivo en Notas a los Estados Financieros en el período 2014

\begin{tabular}{|l|c|c|}
\hline \multicolumn{1}{|c|}{ Contenido } & Frecuencia & $\begin{array}{c}\text { Relación sobre Total de Notas de } \\
\text { Políticas Contables observados } \\
\text { Diciembre 2014 (N=41) }\end{array}$ \\
\hline $\begin{array}{l}\text { Número de empresas que incluyen nota explicativa } \\
\text { sobre política contable del Efectivo }\end{array}$ & 35 & $85.36 \%$ \\
\hline $\begin{array}{l}\text { Número de empresas que no incluyen nota explicativa } \\
\text { sobre política contable del Efectivo }\end{array}$ & 6 & $14.64 \%$ \\
\hline
\end{tabular}

Fuente: Elaboración Propia.

Respecto a las empresas que no han cumplido, debo precisar que todas estas empresas si presentan información detallada de los componentes del efectivo con revelación suficiente.

\section{Análisis a la Categoría de Tipo 4}

La categoría de tipo 4 está definida como las omisiones en la descripción en estados financieros o notas explicativas sobre los componentes del efectivo, las transacciones no monetarias que no se presentan en el Estado de Flujos de Efectivo vinculados a la actividad de inversión y financiación, y el grado de disponibilidad o restricción que tiene el efectivo, que son exigidos por la NIC 7. En ese sentido, de la información presentada en el Cuadro $\mathrm{N}^{\circ} 07$ presenta las omisiones en la revelación de partidas o explicaciones del concepto en notas a los estados financieros. Respecto a revelar los componentes que integran el efectivo el nivel cumplimiento es del $80.49 \%$ ( 8 empresas incumplen lo requerido), respecto a las transacciones no monetarias el nivel de cumplimento es del $46.34 \%$ ( 22 empresas incumplen lo requerido), y finalmente la revelación del nivel de disponibilidad o restricción que tiene el efectivo es del 70.73\% (12 empresas incumplen lo requerido).

Cuadro No 07: Cumplimiento en la Revelación de Componentes y Restricciones del Efectivo en

Notas a los Estados Financieros en el período 2014

\begin{tabular}{|l|r|r|r|r|r|}
\hline \multicolumn{1}{|c|}{ Contenido } & $\begin{array}{c}\text { Intereses } \\
\text { Cobrados }\end{array}$ & $\begin{array}{c}\text { Intereses } \\
\text { Pagados }\end{array}$ & $\begin{array}{c}\text { Dividendos } \\
\text { Cobrados }\end{array}$ & $\begin{array}{c}\text { Dividendos } \\
\text { Pagados }\end{array}$ & $\begin{array}{c}\text { Impuesto } \\
\text { Ganancias } \\
\text { Pagados }\end{array}$ \\
\hline Número de empresas analizadas & 41 & 41 & 41 & 41 & 41 \\
\hline $\begin{array}{l}\text { Número de empresas que reportan la partida o } \\
\text { revelan el concepto }\end{array}$ & 33 & 19 & 29 & 30 & 40 \\
\hline $\begin{array}{l}\text { Número de empresas que no reportan la partida } \\
\text { o el concepto }\end{array}$ & 8 & 22 & 12 & 11 & 1 \\
\hline $\begin{array}{l}\text { Relación de cumplimiento de la revelación } \\
\text { contable }\end{array}$ & $80.49 \%$ & $46.34 \%$ & $70.73 \%$ & 29 & 17 \\
\hline
\end{tabular}

Fuente: Elaboración Propia.

Respecto al resultado obtenido de las revelaciones sobre las transacciones no monetarias, es decir transacciones que no generan movimiento de efectivo pero que por su naturaleza, relevancia y materialidad debe revelarse en el Estado de Flujos de Efectivo o en notas a los estados financieros, si bien es cierto, el resultado mostrado es el de un alto nivel de incumplimiento, esta cifra no es concluyente y definitiva debido a la limitación en acceso de información o la no revelación de las empresas que no incurrieron en transacciones no monetarias. Este comentario es pertinente porque puede presentarse situaciones en 
que la empresa no tiene transacciones no monetarias o teniéndolas a su juicio y mejor criterio no requiere revelación contable.

\section{CONCLUSIONES}

1. Las empresas industriales que cotizan en la Bolsa de Valores de Lima deben preparar información financiera bajo Normas Internacionales de Información Financiera desde el período 2011, año en que se fijó la adopción este modelo normativo, al respecto, dichas normas exigen que las empresas cuyos estados financieros cumplen con las NIIF efectúen en las notas una declaración, explícita y sin reservas de dicho cumplimiento. Por lo indicado es imprescindible tener un conocimiento adecuado de las regulaciones contables vinculadas a la presentación del Estado de Flujos de Efectivo y de las revelaciones contables de los flujos de efectivo del período.

2. De las 41 empresas industriales observadas que reportaron información financiera a la Superintendencia del Mercado de Valores presentaron Estados de Flujos de Efectivo en el período 2014 eligiendo el método directo 34 empresas ( $82.93 \%$ del total) y el método indirecto 7 empresas (17.07\% del total).

3. De la muestra obtenida que alcanza a 41 empresas industriales, y producto del análisis comparativo entre lo requerido por la NIC 7 y el análisis de contenido de la información financiera en la que se valida la partida incurrida, se obtuvo que la relación de cumplimiento de la revelación contable de la partida sobre la partida incurrida en los intereses cobrados es del $22.22 \%$, en los intereses pagados es del 58.54\%, en los dividendos cobrados es del $90.00 \%$, en los dividendos pagados es del $96.67 \%$, $\mathrm{y}$ en los impuestos a las ganancias pagados es del $42.50 \%$ de las empresas. Esta situación nos indica que los lineamientos en la preparación de información financiera sobre los flujos de efectivo contiene insuficiencia informativa que afecta la utilidad de la información en el proceso de toma de decisiones económicas.

4. De las 41 empresas industriales observadas se pudo constatar que existe un alto grado de cum- plimiento de la NIC 7 al revelar los criterios y políticas contables del efectivo y equivalentes de efectivo, cumpliendo lo requerido 35 empresas ( $85.36 \%$ del total). Respecto a las revelaciones contables de los componentes del efectivo, cumplen lo requerido 33 empresas ( $80.49 \%$ del total), y de la disponibilidad o restricción del efectivo, cumplen lo requerido 29 empresas $(70.73 \%$ del total). Es necesario que se logre el nivel de cumplimiento en un $100 \%$ o se indique los motivos por los cuales no se puede cumplir con el requerimiento normativo.

\section{REFERENCIAS BIBLIOGRÁFICAS}

1. ÁlVAREZ,A. (2007) Gestión Contable Bajo US GAAP, Alfaomega Grupo Editor, México.

2. BAUTISTA, C. (2011) Proceso de la Investigación Cualitativa. Epistemología, metodología y aplicaciones, Editorial El Manual Moderno, Colombia.

3. Bolsa de Valores de Lima (2015) Información financiera de empresas cotizantes en el mercado bursátil, disponible en la web www.bvl.com.pe/ mercempresas.html

4. Financial Accounting Standards Board FASB (1987) Statement of Financial Accounting Standards 95, Cash Flow Statement. Estados Unidos.

5. GARCÍA, I. (2013) Introducción a las Normas Internacionales de información Financiera, Alfaomega Grupo Editor, México.

6. International Accounting Standards Board IASB (2015) NIC 7 Estado de Flujos de Efectivo. Inglaterra.

7. KPMG International Financial Reporting Group (2012) Las NIIF comentadas, Editorial Aranzadi S.A., España.

8. The Institute of Chartered Accountants in England and Wales (2011) International Financial Reporting Standards Certificate Manual, Malta.

9. VAN GREUNING, H. (2005) Estándares Internacionales de Información Financiera (2005), Banco Mundial en coedición con Mayol Ediciones S.A., Colombia. 